\title{
Estudo comparativo dos efeitos dento-esqueléticos maxilares e mandibulares da expansão de maxila cirurgicamente assistida
}

\author{
Comparative study of maxillary and mandibular dento-skeletal effects of surgically assisted \\ maxillary expansion
}

Estudio comparativo de los efectos dentoesqueléticos maxilares y mandibulares de la expansión maxilar asistida quirúrgicamente

\section{Resumo}

A expansão rápida de maxila assistida cirurgicamente (ERMAC) é uma das formas de tratamento para deficiência transversal de maxila. Essa técnica depende do uso de dispositivos expansores, os quais podem ter relação com a movimentação dos dentes superiores, não existindo estudos que apontem mudanças dos dentes inferiores. O presente trabalho teve como objetivo mensurar o movimiento produzidos pelo ERMAC, por meio de técnicas cirúrgicas com diferentes tipos de corticotomias da parede anterior da maxila. O estudo foi composto 87 exames de tomografia computadorizada por feixe cônico (TCFC), sendo esses divididos de acordo com a osteotomia realizada: Grupo I ( $\mathrm{n}=$ 42) osteotomia do tipo Le Fort I subtotal com degrau no pilar zigomático-maxilar e Grupo $2(n=45)$ osteotomia do tipo Le Fort I subtotal com osteotomia linear descendente. Os períodos tomados foram divididos em: pré-operatório (T0), após o término da ativação do dispositivo expansor (T1) e contenção ortodôntica (T2). Os dados foram tabulados, comparados entre os períodos estudados e imposto estatisticamente por meio da análise de variância para medidas repetidas (ANOVA) e Teste de Tukey para comparação entre os três tempos, nos quais foram obtidos o total de pacientes (GI + GII) e cada grupo isoladamente (GI) (GII). Os resultados demonstraram aumento estatisticamente significativo das dimensões maxilares; observaram-se efeitos dentoesqueléticos mandibulares na largura da cortical lingual, aumento da distância entre os ápices dos dentes 46 e 36 e grade vestibular do dente 36. Porém, somente esse último foi estatisticamente significativo ( $\mathrm{p}<0,05)$.

Palavras-chave: Mandíbula; Maxila; Técnica de Expansão Palatina; Tomografia Computadorizada por Raios X.

\section{Abstract}

Surgically assisted rapid maxillary expansion (SARME) is the main treatment option for transverse maxillary deficiency in adults. This technique depends on the use of expanders, which are commonly related to the movement of teeth in the maxilla, however, there are no studies analyzing the occurrence of alterations in the lower teeth to this date. The aim of this study was to assess the movement of upper and lower teeth produced by SARME executed by means of two surgical techniques with different types of corticotomies on the anterior wall of the maxilla. The sample consisted of 87 cone beam computed tomography (CBCT) scans, which were divided in two groups according to the osteotomy performed: Group I $(n=42)$ Le Fort I subtotal osteotomy with a step on the zygomatic-buttress and Group 
$2(\mathrm{n}=45)$ Le Fort I type subtotal osteotomy with a linear descending osteotomy of the maxilla. The time periods evaluated were divided into preoperative (T0), immediately after activation of the expander device was ceased (T1) and ending of orthodontic containment period (T2). The data were tabulated and compared between the periods studied and statistically analyzed by means of analysis of variance for repeated measurements (ANOVA) and Tukey's test for comparison between the three time periods. The total number of individuals (GI + GII) and each isolated group (GI) (GII) were analyzed. The results showed a statistically significant increase of all the maxillary dimensions associated with buccal inclination; mandibular dentoskeletal effects on thewidth of the lingual cortex were observed, there was also an increased distance between the apexes of teeth 46 and 36, and buccal inclination of thetooth 36 were noted, however, only the latter was statistically significant $(\mathrm{p}<0.05)$.

Keywords: Mandible; Maxilla; Palatal Expansion Technique; Tomography, X-Ray Computed.

\section{Resumen}

La expansión maxilar rápida asistida quirúrgicamente (SCRAM) es una forma de tratamiento para la deficiencia transversal del maxilar. Esta técnica se basa en el uso de dispositivos de expansión, que pueden estar relacionados con el movimiento de los dientes superiores, pero no hay estudios que apunten a cambios en los dientes inferiores. El propósito de este estudio fue medir el movimiento dental maxilar y mandibular producido por el ERMAC, utilizando técnicas quirúrgicas con diferentes tipos de corticotomías de la pared anterior del maxilar. El estudio comprendía 87 tomografías computarizadas de haz cónico $(\mathrm{CBCT})$, que se dividieron según la osteotomía realizada: Grupo I $(\mathrm{n}=42)$ osteotomía subtotal de Le Fort I con escalón en el pilar cigomático-maxilar y Grupo 2 (n = 45) osteotomía subtotal de Le Fort I con osteotomía lineal descendente. Los periodos tomados se dividieron en: preoperatorio (T0), tras la finalización de la activación del dispositivo expansor (T1) y periodo de retención ortodóntica (T2). Los datos se tabularon, se compararon entre los periodos estudiados y se impusieron estadísticamente mediante el análisis de la varianza para medidas repetidas (ANOVA) y la prueba de Tukey para la comparación entre los tres tiempos, en los que se obtuvo el total de pacientes (GI + GII) y cada grupo solo (GII). Los resultados mostraron un aumento estadísticamente significativo de las dimensiones maxilares, en ambos grupos. Los efectos dentoesqueléticos mandibulares se observaron en la anchura cortical lingual, el aumento de la distancia entre los apices de los dientes 46 y 36 y la gran superficie bucal del diente 36. Sin embargo, sólo este último fue estadísticamente significativo (p < 0,05).

Palabras clave: Mandíbula; Maxilar; Técnica de Expansión Palatina; Tomografía Computarizada de Rayos X.

\section{Introdução}

A dimensão transversal adequada da maxila é um componente fundamental para uma oclusão estável e funcional (Silverstein \& Quinn, 1997). A deficiência maxilar transversa (DMT) representa uma situação comum nos indivíduos portadores de deformidades dento-esqueléticas (Sygouros et al., 2014; Betts, 2016; Bezerra, 2014), ocorrendo em 9,4\% dos casos de mordida cruzada posterior (Bezerra, 2014; Vandersea et al., 2007) e em 30\% dos indivíduos que procuram por cirurgia ortognática (Bezerra, 2014; Proffit et al., 1996). Clinicamente é possível observar a presença de: atresia maxilar, mordida cruzada posterior unilateral ou bilateral, palato ogival, apinhamento e rotação dentais, além de vestibularização dos dentes superiores e inclinação lingual dos dentes inferiores (Vandersea et al, 2007; Betts et al., 1995; Gijt et al., 2017; Assis et al., 2013; Gonçales, 2011). A etiologia da DMT apresenta-se multifatorial, envolvendo fatores de desenvolvimento congênitos, traumáticos e hábitos parafuncionais (Vandersea et al, 2007; Betts et all, 1995; Melsen, 1972; Koudstaal et al., 2005).

O diagnóstico da DMT deve diferenciar a presença de uma deficiência transversal relativa ou absoluta (Vandersea et al, 2007; Cureton \& Cuenin, 1999), sendo que esta última representa uma deficiência transversal verdadeira, com indicação de expansão do arco maxilar (Koudstaal et al, 2005; Chrcanovic \& Custódio, 2009; Lagravère et al., 2006). Seu tratamento consiste no aumento transversal entre as maxilas (Koudstaal et al, 2005; Lagravère et al, 2006) por meio de mecânica ortodôntica, ortopédica e/ou ortopédica-cirúrgica (Betts et al, 1995; Koudstaal et al, 2005; Cureton \& Cuenin, 1999; Assis et al., 2014).

Nos indivíduos que tenham alcançado a maturidade esquelética, o tratamento é preferencialmente realizado por meio da expansão de maxila assistida cirurgicamente (ERMAC) (Betts, 2016; Vandersea et al, 2007; Betts et al, 1995; Gonçales, 2011; Cureton \& Cuenin, 1999; Chrcanovic \& Custódio, 2009; Lagravère et al, 2006; Assis et al, 2014; Bays \& Greco, 1992), uma vez que a consolidação das suturas do crânio e da face resultam em resistência para o movimento expansivo das maxilas 
(Silverstein et al, 1997; Sygouros et al, 2014; Koudstaal et al, 2005; Assis et al, 2014; Bays \& Greco, 1992 ), o que pode resultar em dor, compressão periodontal, extrusão dentária, fenestração da cortical vestibular, necrose da mucosa palatina, reabsorção radicular, recidiva transversal, deslocamento dentário, pouco/nenhuma expansão esquelética e deslocamento do osso alveolar (Gonçales, 2011; Melsen, 1972; Koudstaal et al, 2005; Cureton \& Cuenin, 1999; ; Assis et al, 2014 Bays \& Greco, 1992; Bell \& Epker, 1976; Suri \& Taneja, 2008).

A ERMAC consiste na realização de osteotomias, tornando possível minimizar as áreas de resistência para a expansão maxilar durante a ativação do aparelho expansor (Betts et al, 1995; Assis et al, 2013; Koudstaal et al, 2005; Chrcanovic \& Custódio, 2009; Assis et al, 2014; Suri \& Taneja, 2008; Shetty et al., 1994). No entanto, a literatura apresenta diversos estudos que demonstram a transmissão e dissipação de forças para o tecido ósseo e dentes de suporte (Assis et al, 2013; Assis et al, 2014; Starnbach et al, 1976). Embora seja mais comum a movimentação dos dentes superiores (Vandersea et al, 2007; Chrcanovic \& Custódio, 2009; Lagravère et al, 2006; Assis et al, 2014; Starnbach et al, 1976; Chung \& Goldman, 2014; Oliveira et al., 2016; Bishara \& Staley, 1987) após a expansão de maxila, é possível ocorrer também a movimentação dos dentes inferiores (Lagravère et al, 2006; Oliveira et al, 2016; Bishara \& Staley, 1987; McNamara, 2000; Günbay et al., 2008; Atac Karasu \& Aytac, 2006).

Desta forma, o objetivo deste trabalho foi avaliar a movimentação dos dentes superiores e inferiores, produzidas pela ERMAC, decorrentes da osteotomia do tipo Le Fort I subtotal com dois desenhos diferentes de corticotomias na parede anterior da maxila, sendo 01 com degrau e outro sem degrau.

\section{Metodologia}

O presente trabalho consistiu em uma análise retrospectiva dos exames de tomografia computadorizada por feixe cônico (TCFC) de indivíduos portadores de DMT e tratados por meio de ERMAC. Os exames foram obtidos à partir do banco de dados do Departamento de Cirurgia, Estomatologia, Patologia e Radiologia da Faculdade de Odontologia de Bauru (FOBUSP), após a sua aprovação pelo Comitê de Ética em Pesquisa da Faculdade de Odontologia de Bauru - Universidade de São Paulo (processo de n⿳0 169/2009, CAAE: 43980615.9.0000.5417).

Para esse estudo foram selecionados os exames dos indivíduos submetidos a ERMAC, sendo que esses deveriam ter: prontuários preenchidos corretamente, dados sobre o dispositivo expansor, ritmo de ativação, tipo de osteotomia e controles pós-operatórios. Os exames de TCFC foram avaliados em três tempos distintos: pré-operatório (T0), após o término da expansão (T1) e após a fase de contenção ortodôntica (T2).

De acordo com o desenho da osteotomia realizada na parede anterior da maxila, os grupos foram divididos em: Grupo I (GI), os quais apresentavam um degrau na região do pilar zigomático-maxilar e o Grupo II (GII), nas quais foram realizadas apenas uma osteotomia linear descendente. Para os dois grupos a técnica cirúrgica envolvia também separação da sutura palatina mediana e da placa pterigóide do osso esfenóide. Os arquivos DICOM (Digital Imaging and Communications in Medicine) foram importados para o software Doplhin Imaging® (versão 11.95, Management Solutions, Chatsworth, Califórnia, EUA) e analisados nas reformatações axial e coronal. A avaliação das alterações dento-esqueléticas foi realizada tomando-se por base o estudo de Bezerra ${ }^{4}$. As medidas foram realizadas após a padronização da posição da imagem nos planos anatômicos de acordo com pontos cefalométricos pré-estabelecidos (Figura 1).

O grupo I foi composto por 42 exames de TCFC de 14 indivíduos ( 9 mulheres e 5 homens), com idade média de 25,03 anos. Destes, 8 utilizaram aparelho expansor do tipo Hyrax e 6 com Hass. A fase ortodôntica teve início no $7^{\circ}$ dia pósoperatório, sendo as ativações realizadas com um quarto de volta $(0,25 \mathrm{~mm}), 2$ vezes por dia, resultando em $0,5 \mathrm{~mm}$ de expansão por dia. O protocolo consistiu em uma semana de ativação intercaladas por uma semana de descanso, até que as 
cúspides palatinas dos molares e pré-molares superiores estivessem em contato com as vertentes triturantes das cúspides vestibulares dos dentes inferiores posteriores, sendo essa etapa realizada com uma ligeira sobre correção. Nesse momento, os dispositivos expansores eram estabilizados e deixados em posição por um período de 4 a 6 meses, sendo nesse momento realizado o último exame de imagem (T2). A aquisição das tomografias foi realizada por meio do protocolo de aquisição estendido, com FOV de 0,3mm e 28,45mA, no aparelho I-Cat Classic ${ }^{\circledR}$ (Imaging Sciences, Hatfield, Pensilvânia - EUA).

O grupo II foi composto de 45 exames de TCFC de 15 indivíduos adultos ( 9 do gênero feminino e 6 do gênero masculino), com idade média de 30,2 anos.-Todos foram tratados com o uso de um aparelho expansor palatino do tipo Hyrax fixado aos pré-molares e primeiros molares maxilares. $\mathrm{O}$ início da ativação ocorreu no $7^{\circ}$ dia pós-operatório, sendo realizadas ativações contínuas com um quarto de volta $(0,25 \mathrm{~mm}) 3$ vezes ao dia (a cada $8 \mathrm{~h}$ ), resultando em $0,75 \mathrm{~mm}$ de expansão por dia. A expansão necessária era obtida com uma ligeira sobre correção (T1). Ao término da expansão, o aparelho era mantido em posição por 4 meses e realizada nova TCFC (T2). Os exames foram realizados no protocolo de aquisição estendido FOV de 0,3mm, 28,45mA também no aparelho I-Cat Classic ${ }^{\circledR}$ (Imaging Sciences, Hatfield, Pensilvânia - EUA).

No plano coronal, os caninos $(13,23,33$ e 43$)$ e os primeiros molares $(16,26,36$ e 46) foram utilizados como referência para a realização das mensurações. Todas as análises coronais foram feitas em cortes que permitiam a adequada visualização do longo eixo dos caninos e das raízes dos primeiros molares (palatina para os superiores e canal lingual da raiz mésio-vestibular para o molar inferior) bilateralmente. Na reformatação axial, os cortes foram padronizados, passando através da porção central das placas palatinas e parafuso do aparelho expansor para a total visualização do mesmo.

A avaliação dos efeitos dento-esqueléticos da ERMAC na arcada superior e inferior foi realizada por meio de pontos dentários e ósseos anteriores (nível dos caninos) e posteriores (ao nível dos primeiros molares), sendo possível mensurar: quantidade de expansão, inclinação dentária dos superiores e inferiores e a quantidade de expansão das bases ósseas e alveolares.

Inicialmente foi realizada a reorientação das imagens, tendo a linha édia facial centralizada e o plano sagital determinado pelo alinhamento dos pontos násio (N) e espinha nasal anterior (ANS). Em seguida, o plano axial foi orientado passando pelo rebordo infra-orbitário direito (ORB) e pelo pório $(\mathrm{P})$, paralelamente ao plano horizontal de Frankfurt. Por último, o plano coronal foi posicionado imediatamente posterior ao meato acústico externo direito (Figura 1). A avaliação das alterações ósseas e dentárias transversais foi realizada por meio de medidas realizadas na região posterior no nível dos molares (Figura 2) e anterior no nível dos caninos (Figura 3). A mesma metodologia foi utilizada para avaliar os efeitos da ERMAC sobre os dentes inferiores, ao nível dos dentes anteriores e posteriores. As medidas realizadas foram divididas em de acordo com o Quadro 1. 
Quadro 1: Siglas e descrições das medidas realizadas.

\begin{tabular}{|c|c|}
\hline Medidas & Descrição \\
\hline $\begin{array}{l}\text { LMx } 13-23 \\
\text { LMx } 16-26\end{array}$ & $\begin{array}{l}\text { Largura maxilar, distância entre os pontos mais vestibulares na cortical vestibular imediatamente } \\
\text { acima das raízes dos caninos (13-23) e da raíz mésio vestibular do primeiro molar superior (16-26) }\end{array}$ \\
\hline $\begin{array}{l}\text { LMd 33-43 } \\
\text { LMd 36-46 }\end{array}$ & $\begin{array}{l}\text { Largura mandibular, distância entre os pontos mais vestibulares na cortical vestibular imediatamente } \\
\text { abaixo das raízes dos caninos (33-43) e da raíz mésio vestibular do primeiro molar inferior (36-46) }\end{array}$ \\
\hline $\mathrm{LP}(13,23,16-26,33-43,36-46)$ & $\begin{array}{l}\text { Largura do palato, distância entre os pontos mais internos do osso palatino, imediatamente acima do } \\
\text { ápice da raiz palatina (16-26) e da raíz dos caninos (13-23). } \\
\text { Largura lingual da mandíbula, distância entre os pontos mais internos da cortical lingual da } \\
\text { mandíbula }(33-43,36-46) \text {. }\end{array}$ \\
\hline $\begin{array}{l}\text { DCP }(13-23,16-26) \\
\text { DCP }(33-43,36-46)\end{array}$ & $\begin{array}{l}\text { Distância entre as corticais palatinas do processo alveolar da maxilar/mandibular. } \\
\text { 16-26,46-46: Na região do primeiro molar, na altura do terço médio da raíz palatina. } \\
\text { 13-23,33-43: Na região dos caninos, na altura do ápice dentário. }\end{array}$ \\
\hline $\begin{array}{l}\text { DCV }(13-23,16-26) \\
\text { DCV }(33-43,36-46)\end{array}$ & $\begin{array}{l}\text { Distância entre as corticais vestibulares do processo alveolar maxilares/mandibular direita e esquerda, } \\
\text { na região do primeiro molar, na altura do terço médio da raíz mésio-vestibular. }\end{array}$ \\
\hline AP $(13-23,16-26,33-43,36-46)$ & $\begin{array}{l}\text { Distância entre os ápices dentários das raízes palatinas dos molares (16- } 26 \text { e 36-3) e das raízes dos } \\
\text { caninos (13-23 e 33-43). }\end{array}$ \\
\hline Vest. $(13-23,16-26,33-43,36-46)$ & Distância mésio-vestibular entre o centro das faces vestibulares. \\
\hline Âng (13-23, 16-26, 33-43, 36-46) & $\begin{array}{l}\text { Ângulo formado entre da distância entre os ápices dentários (Ap) com a linha que passa no centro do } \\
\text { longo eixo das raízes palatinas dos molares }(16-26,36-46) \text { e/ou dos caninos (13-23.33-43). }\end{array}$ \\
\hline
\end{tabular}

Fonte: Autores (2021).

Figura 1. Ilustração da padronização dos exames, realizando a reorientação dos planos sagital (linha vermelha), axial coronal (linha azul) e axial (linha verde).
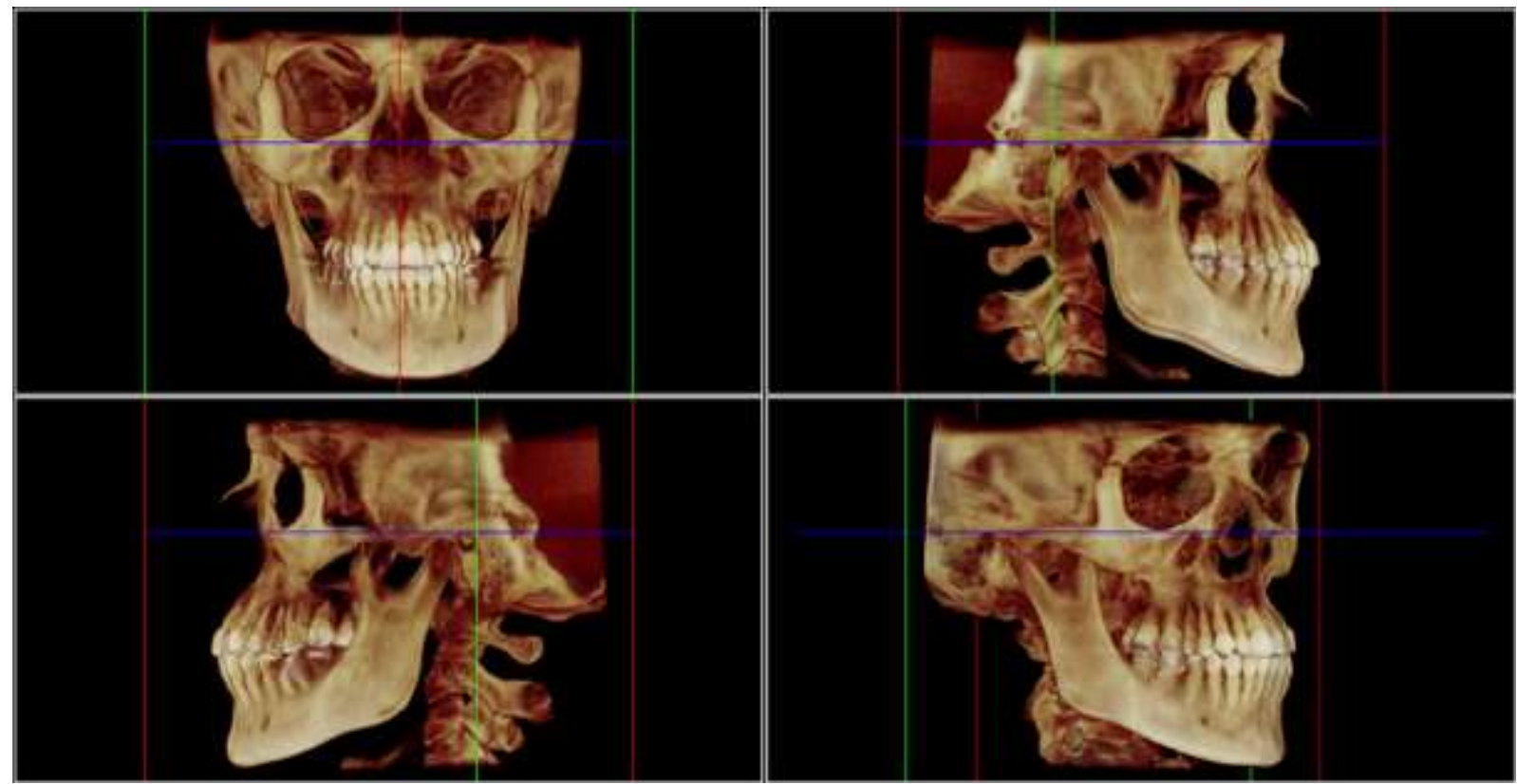

Fonte: Autores (2021). 
Figura 2. Avaliação dos efeitos transversais da ERMCA na região posterior da maxila: LMx, largura da base óssea maxilar; LP, largura do palato; DCP, distância entre as corticais palatinas do processo alveolar; DCV, distância entre as corticais vestibulares do processo alveolar vestibular; Ap., distância entre os ápices dentários; Vest., distância entre as faces vestibulares; e Âng., angulação dos dentes.

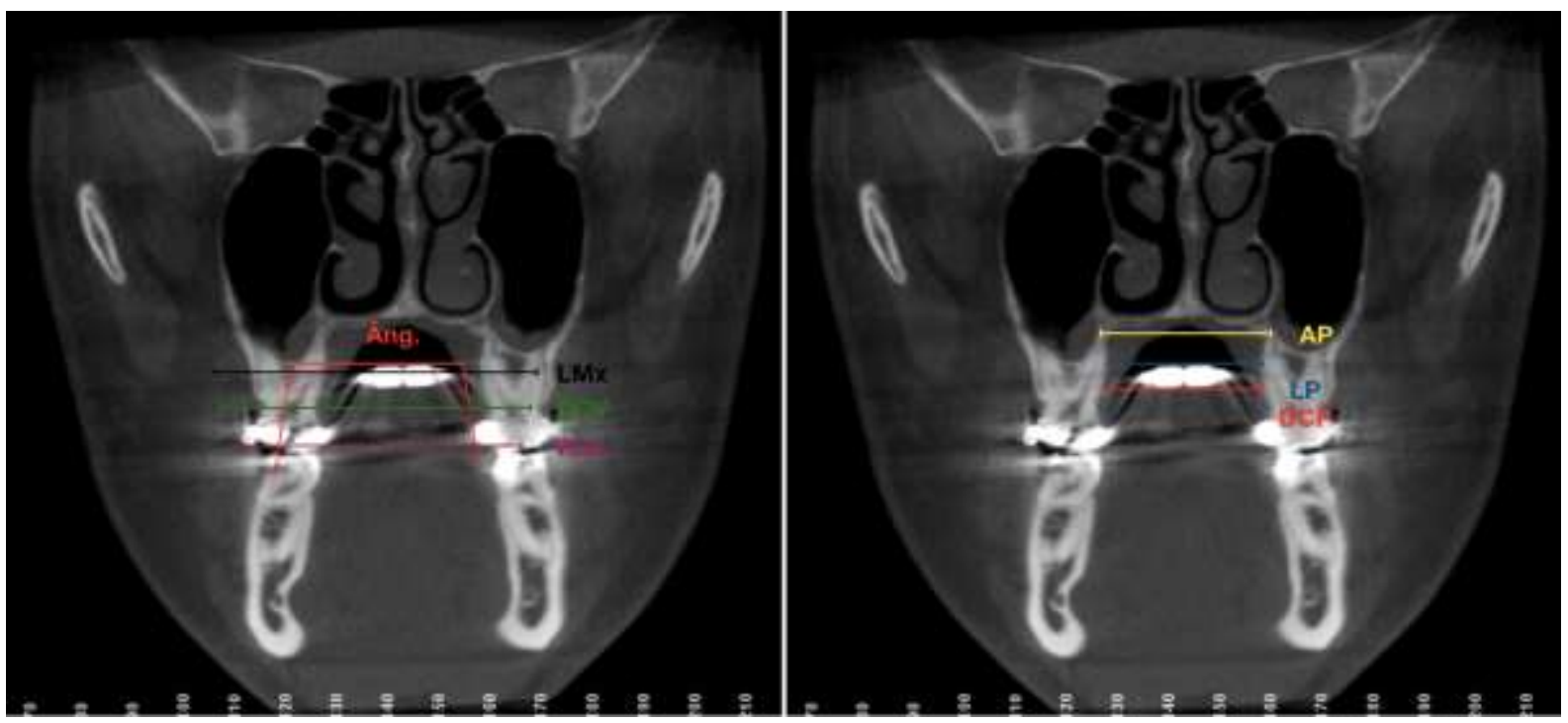

Fonte: Autores (2021).

Figura 3. Avaliação coronal dos efeitos transversais da ERMCA na região anterior da maxila: LMx, largura da base óssea maxilar; LP, largura do palato; DCP, distância entre as corticais palatinas do processo alveolar; DCV, distância entre as corticais vestibulares do processo alveolar vestibular; Ap., distância entre os ápices dentários; Vest., distância entre as faces vestibulares; e Âng., angulação dos dentes.
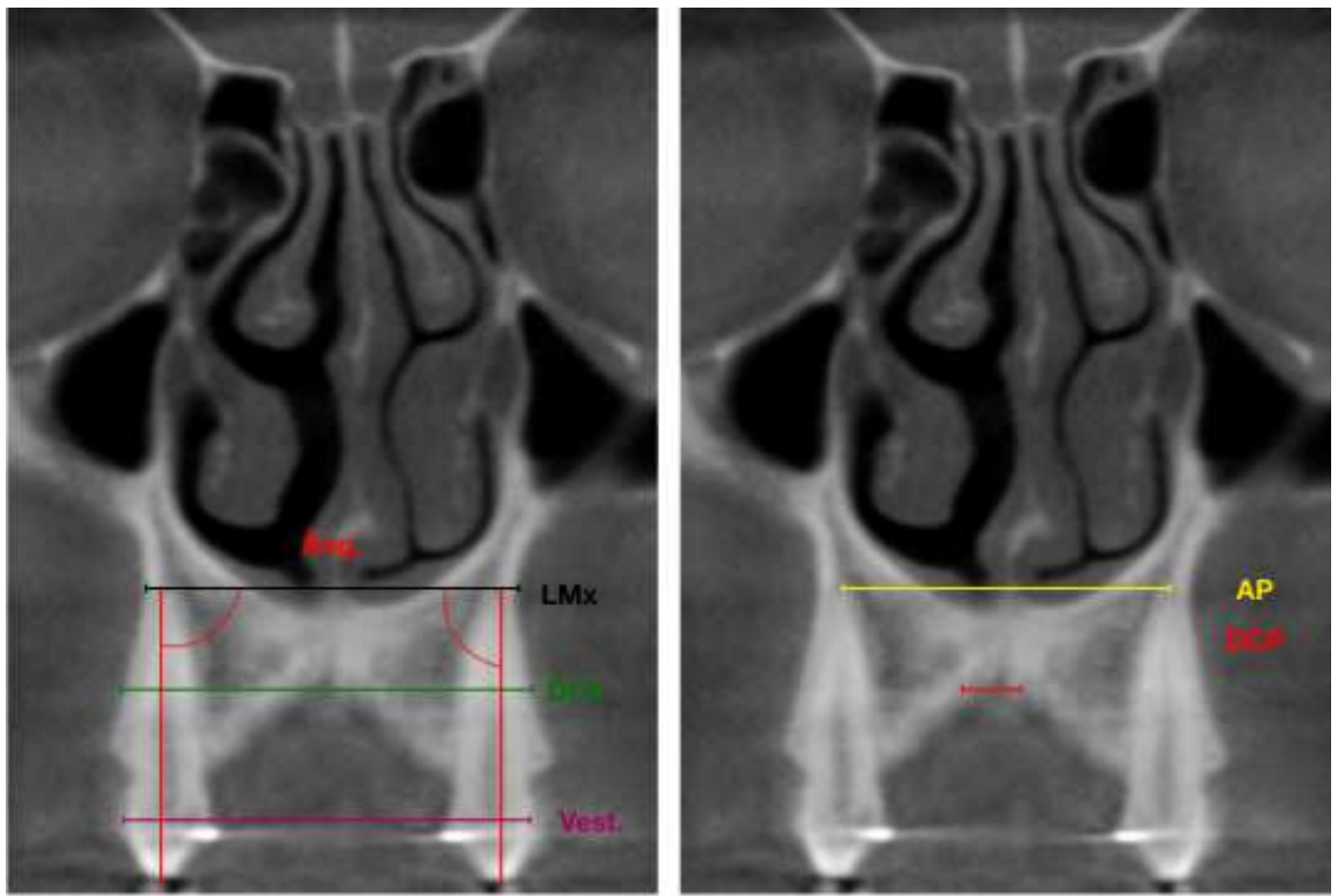

Fonte: Autores (2021). 
Todas as medidas foram realizadas pelo mesmo avaliador. Para que fosse possível a avaliação do erro do método, realizaram-se novas medidas em todas as imagens, para análise do erro casual e sistemático, por meio do Coeficiente de Correlação Intraclasse, com intervalo de pelo menos 15 dias.

Após coleta dos dados, os mesmos foram tabulados, comparados entre os períodos estudados e analisados por meio da análise de variância para medidas repetidas (ANOVA) e teste de Tukey para comparação entre os três tempos, nos quais foram avaliados os grupos isoladamente (GI) e (GII) e o total de indivíduos (GI+GII).

\section{Resultados}

O estudo foi composto por 87 exames de TCFC de 29 indivíduos adultos submetidos à ERMAC, com dois desenhos diferentes de corticotomias na parede lateral da maxila, totalizando 42 tomografias no grupo I - sendo 14 para cada um dos tempos estudados (T0, T1 e T2) e 15 exames para o grupo II. O estudo foi composto por 18 mulheres e 11 homens, com idade média de 27,75 anos (desvio padrão de 6,55 anos).

A avaliação dos efeitos da ERMAC e as alterações nos parâmetros maxilares e mandibulares pré-determinados foram realizadas por meio da mensuração entre os períodos pré-operatório e após o término das ativações do aparelho expansor (T0T1), pré-operatório e o pós-operatório de (T0-T2) e a comparação entre os tempos T1-T2. Os dados foram tabelados considerando as variações observadas nas regiões anteriores e posteriores da maxila e da mandíbula. Os parâmetros que foram estatisticamente significantes para o teste de variância para medidas repetidas (ANNOVA) foram submetidos ao teste de Tukey, sendo possível determinar em quais momentos as alterações apresentaram significância estatística.

A análise do erro pelo Coeficiente de Correlação inter-classe (CCI), apresentou-se excelente, uma vez que todos seus valores foram superiores a 0,75 . Apenas dois parâmetros, em um único indivíduo, apresentaram ICC satisfatório, pois foram inferiores a 0,75 .

\section{Medidas dos grupos I e II em T1}

Com relação a região anterior de maxila, no GI foi possível observar um aumento estatisticamente significante de todos os parâmetros avaliados $(\mathrm{p}<0,05)$. Para o grupo II, não foram observadas alterações estatisticamente significantes apenas das medidas da largura da base óssea maxilar (DV) e da distância dos processos alveolares palatinos.

Com relação a região posterior da maxila, em ambos os grupos, todos os parâmetros aumentaram de maneira estatisticamente significativa $(\mathrm{p}<0,05)$ (Tabelas 1 e 2).

\section{Medidas dos grupos em T2}

Após o período de contenção ortodôntica, as medidas permaneceram maiores do que as observadas em T1, sendo essa diferença estatisticamente significante (Tabelas 1 e 2).

\section{Medidas dos grupos entre os tempos T1 e T2}

Após o término das ativações e estabilização do dispositivo expansor, foi possível realizar a avaliar alterações, em ambos os grupos, observadas entre o período T1 e T2.

Para os indivíduos do grupo I na região anterior observou-se diminuição das medidas LMx, DCP, Vest.13-23, e angulação dos dentes 13 e 23 e aumento das medidas AP e DCV na parte anterior. Já para a região posterior ocorreu aumento das medidas LMx, DCV, AP e VEST 16-26 e diminuição das medidas e das medidas DCP, LP e Âng.16 e Âng.26. Dessas alterações, somente, VEST 13-23, angulação do dente 23 e Ap. 16-26 foram estatisticamente significantes (Tabela 1 e 2). 
Para o grupo II, observou-se aumento na região anterior observou-se aumento das medidas LMx, DCP e AP e diminuição DCV, Âng.13 e Âng.23. Para a região de molares, ocorreu aumento das medidas LMx, DCP, DCV, LP, AP, VEST 16-26 e diminuição de DCV, Âng.16 e Âng.26. Os dentes 13 e 23 não sofreram nenhum tipo de alteração em relação a sua posição vestibular, quando comparados ao tempo T1. Das alterações observadas, somente a distância entre os ápices dos dentes posteriores com significância estatística $(\mathrm{p}<0,05)$.

\section{Comparação das medidas entre os grupos 1 e 2.}

A análise dos valores obtidos pelo teste de Anova e de Tukey apontam que para ambos foi possível observar expansão das maxilas, sendo observado aumento estatisticamente significante dos parâmetros avaliados na comparação entre os tempos T0-T1 e T0-T2.

No GI com relação a região anterior de maxila observou-se aumento significante para as medidas anteriores (DCV, distância ápices, VEST13-23, Âng 13 e 23) e posteriores (LMx, LP, DCV, Ápice, VEST 16-26 e Âng 16 e 26).

A avaliação de T1-T2 aponta para um aumento significante $(\mathrm{p}<0,05)$ da distância entre os ápices na região anterior e posterior da maxila. Os demais paâmetros não apresentaram significância estatística.

Para a região posterior observamos diferença entre a largura do processo alveolar palatino, ocorrendo diminuição no G I e aumento no GII, com diferença estatisticamente significante entre os grupos nos controles de T0-T1 e de T1-T2. A largura do palato aumentou no G II e diminuiu no G I entre os períodos T1-T2, sendo essas medidas estatisticamente significantes $(\mathrm{p}=0,015)$.

Tabela 1. Medidas das alterações $(\mathrm{mm})$ dos parâmetros da região anterior da maxila, nos tempos T0, T1 e T2. Médias (M), desvio padrão (dp), resultado do teste Anova para medidas repetidas e de Tukey, para os dois grupos, utilizando nível de significância de $95 \%(\mathrm{p}<0,05)$.

\begin{tabular}{|c|c|c|c|c|c|c|c|c|}
\hline $\begin{array}{l}\text { Medi } \\
\text { das }\end{array}$ & $\begin{array}{l}\text { Gru } \\
\text { pos }\end{array}$ & $\begin{array}{l}\text { T0 } \\
\text { M (dp) }\end{array}$ & $\begin{array}{l}\text { T1 } \\
\text { M (dp) }\end{array}$ & $\begin{array}{l}\text { T2 } \\
\text { M (dp) }\end{array}$ & $\begin{array}{l}\text { p } \\
\text { (Anova) }\end{array}$ & $\begin{array}{l}\text { T0-T1 } \\
\text { (Tukey) }\end{array}$ & $\begin{array}{l}\text { T1-T2 } \\
\text { (Tukey) }\end{array}$ & $\begin{array}{l}\text { T0-T2 } \\
\text { (Tukey) }\end{array}$ \\
\hline LMx & $\begin{array}{l}\text { G I } \\
\text { G II }\end{array}$ & $\begin{array}{l}32,11(4,44) \\
30,07(2,70)\end{array}$ & $\begin{array}{l}36,64(3,19) \\
33,00(3,29)\end{array}$ & $\begin{array}{l}36,27(3,18) \\
37,67(18,60)\end{array}$ & $\begin{array}{l}0,00^{*} \\
0,22\end{array}$ & $\begin{array}{l}0,00 * \\
0,77\end{array}$ & $\begin{array}{l}0,94 \\
0,52\end{array}$ & $\begin{array}{l}0,00^{*} \\
0,20\end{array}$ \\
\hline DCP & $\begin{array}{l}\text { G I } \\
\text { G II }\end{array}$ & $\begin{array}{l}8(4,530 \\
7,56(5,59)\end{array}$ & $\begin{array}{l}10,64(5,25) \\
8,36(4,30)\end{array}$ & $\begin{array}{l}10,28(4,43) \\
9,54(4,84)\end{array}$ & $\begin{array}{l}0,01^{*} \\
0,37\end{array}$ & $\begin{array}{l}0,01 * \\
0,83\end{array}$ & $\begin{array}{l}0,48 \\
0,67\end{array}$ & $\begin{array}{l}0,14 \\
0,34\end{array}$ \\
\hline DCV & $\begin{array}{l}\text { G I } \\
\text { G II }\end{array}$ & $\begin{array}{l}36,53(1,91) \\
34,52(1,88)\end{array}$ & $\begin{array}{l}41,66(2,39) \\
38,14(2,62)\end{array}$ & $\begin{array}{l}40,98(2,22) \\
38,7(1,90)\end{array}$ & $\begin{array}{l}0,00^{*} \\
0,00^{*}\end{array}$ & $\begin{array}{l}0,00 * \\
0,00 *\end{array}$ & $\begin{array}{l}0,26 \\
0,54\end{array}$ & $\begin{array}{l}0,00^{*} \\
0,00^{*}\end{array}$ \\
\hline $\mathbf{A P}$ & $\begin{array}{l}\text { G I } \\
\text { G II }\end{array}$ & $\begin{array}{l}26,37(2,57) \\
24,01(2,23)\end{array}$ & $\begin{array}{l}30,08(2,11) \\
27,12(2,19)\end{array}$ & $\begin{array}{l}30,77(2,34) \\
27,54(1,99)\end{array}$ & $\begin{array}{l}0,00^{*} \\
0,00^{*}\end{array}$ & $\begin{array}{l}0,00 * \\
0,00 *\end{array}$ & $\begin{array}{l}0,41 \\
0,74\end{array}$ & $\begin{array}{l}0,00^{*} \\
0,00^{*}\end{array}$ \\
\hline $\begin{array}{l}\text { VEST } \\
13-23\end{array}$ & $\begin{array}{l}\text { G I } \\
\text { G II }\end{array}$ & $\begin{array}{l}36,91(3,01) \\
36,45(2,06)\end{array}$ & $\begin{array}{l}43,14(3,43) \\
41,59(3,02)\end{array}$ & $\begin{array}{l}41,12(3,77) \\
41,59(2,17)\end{array}$ & $\begin{array}{l}0,00 * \\
0,00 *\end{array}$ & $\begin{array}{l}0,00^{*} \\
0,00^{*}\end{array}$ & $\begin{array}{l}0,00 * \\
1\end{array}$ & $\begin{array}{l}0,00^{*} \\
0,00^{*}\end{array}$ \\
\hline ÂNG 23 & $\begin{array}{l}\text { G I } \\
\text { GII }\end{array}$ & $\begin{array}{l}94,84(5,49) \\
96,2(4,37)\end{array}$ & $\begin{array}{l}100,72(5,65) \\
100,15(5,15)\end{array}$ & $\begin{array}{l}96,30(5,53) \\
98,62(4,03)\end{array}$ & $\begin{array}{l}0,00^{*} \\
0,00^{*}\end{array}$ & $\begin{array}{l}0,00^{*} \\
0,00^{*}\end{array}$ & $\begin{array}{l}0,07^{*} \\
0,44\end{array}$ & $\begin{array}{l}0,44 \\
0,04^{*}\end{array}$ \\
\hline
\end{tabular}

Fonte: Autores (2021). 
Tabela 2. Medidas das alterações $(\mathrm{mm})$ dos parâmetros da região posterior da maxila, nos tempos T0, T1 e T2. Médias (M), desvio padrão (dp), resultado do teste de Anova para medidas repetidas, e teste Tukey, para os dois grupos, utilizando nível de significância de $95 \%(\mathrm{p}<0,05)$.

\begin{tabular}{|c|c|c|c|c|c|c|c|c|}
\hline $\begin{array}{l}\text { Medi } \\
\text { das }\end{array}$ & $\begin{array}{l}\text { Gru } \\
\text { pos }\end{array}$ & $\begin{array}{l}\text { T0 } \\
M(d p)\end{array}$ & $\begin{array}{l}T 1 \\
M(d p)\end{array}$ & $\begin{array}{l}T 2 \\
M(d p)\end{array}$ & $p$ (Anova) & $\begin{array}{l}\text { T0-T1 } \\
\text { (Tukey) }\end{array}$ & $\begin{array}{l}\text { T1-T2 } \\
\text { (Tukey) }\end{array}$ & $\begin{array}{l}\text { T0-T2 } \\
\text { (Tukey) }\end{array}$ \\
\hline$L M x$ & $\begin{array}{l}\text { G I } \\
\text { G II }\end{array}$ & $\begin{array}{l}57,68(4,52) \\
51,40(4,66)\end{array}$ & $\begin{array}{l}59,07(3,59) \\
54,00(3,90\end{array}$ & $\begin{array}{l}59,18(3,58) \\
54,20(4,21)\end{array}$ & $\begin{array}{l}0,00^{*} \\
0,00^{*}\end{array}$ & $\begin{array}{l}0,00 * \\
0,00 *\end{array}$ & $\begin{array}{l}0,95 \\
0,14\end{array}$ & $\begin{array}{l}0,00^{*} \\
0,00^{*}\end{array}$ \\
\hline$D C P$ & $\begin{array}{l}\text { G I } \\
\text { G II }\end{array}$ & $\begin{array}{l}29,59(3,55) \\
27,57(4,10)\end{array}$ & $\begin{array}{l}34,06(3,75) \\
30,59(4,41)\end{array}$ & $\begin{array}{l}33,31(3,96) \\
31,07(3,00)\end{array}$ & $\begin{array}{l}0,00^{*} \\
0,00^{*}\end{array}$ & $\begin{array}{l}0,00^{*} \\
0,00 *\end{array}$ & $\begin{array}{l}0,07 \\
0,66\end{array}$ & $\begin{array}{l}0,00^{*} \\
0,00^{*}\end{array}$ \\
\hline$D C V$ & $\begin{array}{l}\text { G I } \\
\text { G II }\end{array}$ & $\begin{array}{l}55,53(3,47) \\
55,13(4,46)\end{array}$ & $\begin{array}{l}59,41(3,51) \\
57,88(3,55)\end{array}$ & $\begin{array}{l}59,52(3,48) \\
58,74(3,86)\end{array}$ & $\begin{array}{l}0,00 * \\
0,00 *\end{array}$ & $\begin{array}{l}0,00^{*} \\
0,00^{*}\end{array}$ & $\begin{array}{l}0,93 \\
0,18\end{array}$ & $\begin{array}{l}0,00^{*} \\
0,00^{*}\end{array}$ \\
\hline$L P$ & $\begin{array}{l}\text { G I } \\
\text { G II }\end{array}$ & $\begin{array}{l}24,90(6,36) \\
25,85(5,83)\end{array}$ & $\begin{array}{l}27,87(7,42) \\
28,04(4,41)\end{array}$ & $\begin{array}{l}27,12(7,78) \\
28,38(4,81)\end{array}$ & $\begin{array}{l}0,00^{*} \\
0,00^{*}\end{array}$ & $\begin{array}{l}0,00^{*} \\
0,00^{*}\end{array}$ & $\begin{array}{l}0,14 \\
0,81\end{array}$ & $\begin{array}{l}0,00^{*} \\
0,00^{*}\end{array}$ \\
\hline$A P$ & $\begin{array}{l}\text { G I } \\
\text { G II }\end{array}$ & $\begin{array}{l}33,30(4,29) \\
32,37(6,73)\end{array}$ & $\begin{array}{l}36,95(4,31) \\
36,66(5,13)\end{array}$ & $\begin{array}{l}37,95(4,11) \\
37,49(4,95)\end{array}$ & $\begin{array}{l}0,00^{*} \\
0,00^{*}\end{array}$ & $\begin{array}{l}0,00 * \\
0,00 *\end{array}$ & $\begin{array}{l}0,00^{*} \\
0,00^{*}\end{array}$ & $\begin{array}{l}0,07 \\
0,00^{*}\end{array}$ \\
\hline $\begin{array}{l}V E S T \\
16-26\end{array}$ & $\begin{array}{l}\text { G I } \\
\text { G II }\end{array}$ & $\begin{array}{l}54,09(3,83) \\
52,42(3,86)\end{array}$ & $\begin{array}{l}60,43(4,94) \\
58,94(4,96)\end{array}$ & $\begin{array}{l}60,58(4,67) \\
59,33(4,08)\end{array}$ & $\begin{array}{l}0,00^{*} \\
0,00^{*}\end{array}$ & $\begin{array}{l}0,00^{*} \\
0,00 *\end{array}$ & $\begin{array}{l}0,95 \\
0,86\end{array}$ & $\begin{array}{l}0,00^{*} \\
0,00^{*}\end{array}$ \\
\hline $\begin{array}{l}A N G \\
16\end{array}$ & $\begin{array}{l}\text { G I } \\
\text { G II }\end{array}$ & $\begin{array}{l}99,50(6,58) \\
99,81(7,13)\end{array}$ & $\begin{array}{l}105,66(7,58) \\
105,93(7,72)\end{array}$ & $\begin{array}{l}103,44(9,28) \\
103,36(4,40)\end{array}$ & $\begin{array}{l}0,00^{*} \\
0,00^{*}\end{array}$ & $\begin{array}{l}0,00 * \\
0,00 *\end{array}$ & $\begin{array}{l}0,13 \\
0,19\end{array}$ & $\begin{array}{l}0,00 * \\
0,05\end{array}$ \\
\hline $\begin{array}{l}A N G \\
26\end{array}$ & $\begin{array}{l}\text { G I } \\
\text { G II }\end{array}$ & $\begin{array}{l}102,29(7,32) \\
100,02(8,67)\end{array}$ & $\begin{array}{l}108,50(9,84) \\
104,58(9,50)\end{array}$ & $\begin{array}{l}107,59(9,97) \\
103,40(9,16)\end{array}$ & $\begin{array}{l}0,00^{*} \\
0,00^{*}\end{array}$ & $\begin{array}{l}0,00^{*} \\
0,00^{*}\end{array}$ & $\begin{array}{l}0,71 \\
0,57\end{array}$ & $\begin{array}{l}0,00^{*} \\
0,02^{*}\end{array}$ \\
\hline
\end{tabular}

Fonte: Autores (2021).

Para ambos os grupos, os efeitos mandibulares forem discretos, sendo observada significância estatística somente para a angulação dos dentes 36, em ambos os grupos. No grupo I, essa alteração foi significante nos tempos T0-T1 e T0-T2, enquanto para o grupo II, ela foi significante somente nos tempos T0-T1 (Tabela 4).

Tabela 3. Medidas das alterações $(\mathrm{mm})$ dos parâmetros da região anterior da mandíbula, nos tempos T0, T1 e pós-operatório T2. Médias (M), desvio padrão (dp), resultado do teste Anova para medidas repetidas, para os dois grupos, utilizando nível de significância de $95 \%(\mathrm{p}<0,05)$.

\begin{tabular}{|c|c|c|c|c|c|c|c|c|}
\hline $\begin{array}{l}\text { Medi } \\
\text { das }\end{array}$ & $\begin{array}{l}\text { Gru } \\
\text { pos }\end{array}$ & $\begin{array}{l}\text { T0 } \\
\text { M (dp) }\end{array}$ & $\begin{array}{l}\text { T1 } \\
\text { M (dp) }\end{array}$ & $\begin{array}{l}\text { T2 } \\
\text { M (dp) }\end{array}$ & $\mathbf{p}$ & $\begin{array}{l}\text { T0-T1 } \\
\text { (Tukey) }\end{array}$ & $\begin{array}{l}\text { T1-T2 } \\
\text { (TUkey) }\end{array}$ & $\begin{array}{l}\text { T0-T2 } \\
\text { (Tukey) }\end{array}$ \\
\hline LMd & $\begin{array}{l}\text { G I } \\
\text { G II }\end{array}$ & $\begin{array}{l}35,80(5,15) \\
33,89(4,31)\end{array}$ & $\begin{array}{l}36,08(5,34) \\
34,31(3,88)\end{array}$ & $\begin{array}{l}36,11(5,77) \\
33,40(4,33)\end{array}$ & $\begin{array}{l}0,81 \\
0,81\end{array}$ & $\begin{array}{l}0,86 \\
0,81\end{array}$ & $\begin{array}{l}0,99 \\
0,88\end{array}$ & $\begin{array}{l}0,82 \\
0,99\end{array}$ \\
\hline$D C V$ & $\begin{array}{l}\text { G I } \\
\text { G II }\end{array}$ & $\begin{array}{l}32,93(2,44) \\
32,59(2,97)\end{array}$ & $\begin{array}{l}32,92(2,49) \\
32,37(3,12)\end{array}$ & $\begin{array}{l}33,36(2,69) \\
31,95(2,50)\end{array}$ & $\begin{array}{l}0,37 \\
0,38\end{array}$ & $\begin{array}{l}0,99 \\
0,88\end{array}$ & $\begin{array}{l}0,44 \\
0,63\end{array}$ & $\begin{array}{l}0,45 \\
0,36\end{array}$ \\
\hline$A P$ & $\begin{array}{l}\text { G I } \\
\text { G II }\end{array}$ & $\begin{array}{l}22,86(2,44) \\
22,60(3,47)\end{array}$ & $\begin{array}{l}22,84(2,49) \\
22,69(3,77)\end{array}$ & $\begin{array}{l}22,60(3,11) \\
22,62(3,01)\end{array}$ & $\begin{array}{l}0,57 \\
0,98\end{array}$ & $\begin{array}{l}0,99 \\
0,98\end{array}$ & $\begin{array}{l}0,65 \\
0,99\end{array}$ & $\begin{array}{l}0,61 \\
0,98\end{array}$ \\
\hline $\begin{array}{l}\text { VEST } \\
13-23\end{array}$ & $\begin{array}{l}\text { G I } \\
\text { G II }\end{array}$ & $\begin{array}{l}32,64(2,35) \\
32,52(2,91)\end{array}$ & $\begin{array}{l}32,66(1,98) \\
32,50(2,57)\end{array}$ & $\begin{array}{l}33,72(2,53) \\
33,69(1,20)\end{array}$ & $\begin{array}{l}0,11 \\
0,89\end{array}$ & $\begin{array}{l}0,99 \\
0,99\end{array}$ & $\begin{array}{l}0,17 \\
0,90\end{array}$ & $\begin{array}{l}0,15 \\
0,92\end{array}$ \\
\hline $\begin{array}{l}\hat{A} N G \\
33\end{array}$ & $\begin{array}{l}\text { G I } \\
\text { G II }\end{array}$ & $\begin{array}{l}98,40(7,13) \\
95,80(6,77)\end{array}$ & $\begin{array}{l}94,66(5,92) \\
95,42(6,97)\end{array}$ & $\begin{array}{l}97,47(8,05) \\
96,69(7,24)\end{array}$ & $\begin{array}{l}0,20 \\
0,50\end{array}$ & $\begin{array}{l}0,99 \\
0,93\end{array}$ & $\begin{array}{l}0,28 \\
0,48\end{array}$ & $\begin{array}{l}0,24 \\
0,69\end{array}$ \\
\hline $\begin{array}{l}\hat{A} N G \\
43\end{array}$ & $\begin{array}{l}\text { G I } \\
\text { GII }\end{array}$ & $\begin{array}{l}94,34(6,46) \\
95,34(3,60)\end{array}$ & $\begin{array}{l}94,46(7,22) \\
95,37(4,25)\end{array}$ & $\begin{array}{l}96,32(9,53) \\
94,70(4,38)\end{array}$ & $\begin{array}{l}0,23 \\
0,60\end{array}$ & $\begin{array}{l}0,98 \\
0,99\end{array}$ & $\begin{array}{l}0,34 \\
0,68\end{array}$ & $\begin{array}{l}0,26 \\
0,62\end{array}$ \\
\hline
\end{tabular}

Fonte: Autores (2021). 
Tabela 4. Medidas das alterações $(\mathrm{mm})$ dos parâmetros da região posterior da mandíbula, nos tempos T0, T1 e pós-operatório T2. Médias (M), desvio padrão (dp), resultado do teste Anova para medidas repetidas, para os dois grupos, utilizando nível de significância de $95 \%(\mathrm{p}<0,05)$.

\begin{tabular}{|c|c|c|c|c|c|c|c|c|}
\hline $\begin{array}{l}\text { Medi } \\
\text { das }\end{array}$ & $\begin{array}{l}\text { Gru } \\
\text { pos }\end{array}$ & $\begin{array}{l}\text { T0 } \\
\text { M(dp) }\end{array}$ & $\begin{array}{l}\text { T1 } \\
\text { M (dp) }\end{array}$ & $\begin{array}{l}\text { T2 } \\
M(d p)\end{array}$ & $\mathbf{p}$ & $\begin{array}{l}\text { T0-T1 } \\
\text { (Tukey) }\end{array}$ & $\begin{array}{l}\text { T1-T2 } \\
\text { (Tukey) }\end{array}$ & $\begin{array}{l}\text { T0-T2 } \\
\text { (Tukey) }\end{array}$ \\
\hline LMd & $\begin{array}{l}\text { G I } \\
\text { G II }\end{array}$ & $\begin{array}{l}65,00(8,85) \\
68,40(6,45)\end{array}$ & $\begin{array}{l}64,31(9,14) \\
68,01(7,47)\end{array}$ & $\begin{array}{l}63,85(9,52) \\
67,50(6,510\end{array}$ & $\begin{array}{l}0,09 \\
0,77\end{array}$ & $\begin{array}{l}0,36 \\
0,94\end{array}$ & $\begin{array}{l}0,64 \\
0,91\end{array}$ & $\begin{array}{l}0,08 \\
0,75\end{array}$ \\
\hline$D C P$ & $\begin{array}{l}\text { G I } \\
\text { G II }\end{array}$ & $\begin{array}{l}36,00(5,25) \\
37,40(4,96)\end{array}$ & $\begin{array}{l}35,48(5,67) \\
37,02(5,22)\end{array}$ & $\begin{array}{l}35,42(5,71) \\
36,70(5,06)\end{array}$ & $\begin{array}{l}0,38 \\
0,10\end{array}$ & $\begin{array}{l}0,48 \\
0,45\end{array}$ & $\begin{array}{l}0,99 \\
0,57\end{array}$ & $\begin{array}{l}0,43 \\
0,08\end{array}$ \\
\hline$D C V$ & $\begin{array}{l}\text { G I } \\
\text { G II }\end{array}$ & $\begin{array}{l}58,75(7,30) \\
62,97(6,03)\end{array}$ & $\begin{array}{l}58,72(8,82) \\
62,43(6,550\end{array}$ & $\begin{array}{l}58,37(8,30) \\
61,26(, 61)\end{array}$ & $\begin{array}{l}0,60 \\
0,09\end{array}$ & $\begin{array}{l}0,99 \\
0,75\end{array}$ & $\begin{array}{l}0,68 \\
0,29\end{array}$ & $\begin{array}{l}0,63 \\
0,08\end{array}$ \\
\hline$L P$ & $\begin{array}{l}\text { G I } \\
\text { G II }\end{array}$ & $\begin{array}{l}38,28(7,66) \\
40,38(6,14)\end{array}$ & $\begin{array}{l}37,79(8,56) \\
39,65(7,00)\end{array}$ & $\begin{array}{l}37,28(6,62) \\
39,03(4,66)\end{array}$ & $\begin{array}{l}0,11 \\
0,06\end{array}$ & $\begin{array}{l}0,54 \\
0,38\end{array}$ & $\begin{array}{l}0,52 \\
0,50\end{array}$ & $\begin{array}{l}0,09 \\
0,05\end{array}$ \\
\hline$A P$ & $\begin{array}{l}\text { G I } \\
\text { G II }\end{array}$ & $\begin{array}{l}52,50(5,90) \\
54,75(4,66)\end{array}$ & $\begin{array}{l}55,88(5,56) \\
56,29(3,62)\end{array}$ & $\begin{array}{l}56,32(5,23) \\
56,31(3,44)\end{array}$ & $\begin{array}{l}0,07 \\
0,38\end{array}$ & $\begin{array}{l}0,48 \\
0,37\end{array}$ & $\begin{array}{l}0,99 \\
0,92\end{array}$ & $\begin{array}{l}0,43 \\
0,6\end{array}$ \\
\hline $\begin{array}{l}\text { VEST 36- } \\
46\end{array}$ & $\begin{array}{l}\text { G I } \\
\text { G II }\end{array}$ & $\begin{array}{l}55,79(4,61) \\
56,66(3,14)\end{array}$ & $\begin{array}{l}55,88(5,56) \\
56,29(3,62)\end{array}$ & $\begin{array}{l}56,32(5,23) \\
56,31(3,44)\end{array}$ & $\begin{array}{l}0,50 \\
0,46\end{array}$ & $\begin{array}{l}1 \\
0,52\end{array}$ & $\begin{array}{l}0,63 \\
1\end{array}$ & $\begin{array}{l}0,34 \\
0,92\end{array}$ \\
\hline$A N G 36$ & $\begin{array}{l}\text { G I } \\
\text { G II }\end{array}$ & $\begin{array}{l}78,17(7,53) \\
72,94(7,24)\end{array}$ & $\begin{array}{l}81,06(8,89) \\
74,45(6,23)\end{array}$ & $\begin{array}{l}81,58(10,29) \\
73,01(6,31)\end{array}$ & $\begin{array}{l}0,00 * \\
0,02 *\end{array}$ & $\begin{array}{l}0,01 * \\
0,04 *\end{array}$ & $\begin{array}{l}0,85 \\
0,05\end{array}$ & $\begin{array}{l}0,00 * \\
0,991\end{array}$ \\
\hline$A N G 46$ & $\begin{array}{l}\text { G I } \\
\text { GII }\end{array}$ & $\begin{array}{l}76,93(6,67) \\
74,60(5,67)\end{array}$ & $\begin{array}{l}76,90(6,49) \\
74,93(4,76)\end{array}$ & $\begin{array}{l}78,06(8,90) \\
73,98(4,85)\end{array}$ & $\begin{array}{l}0,25 \\
0,83\end{array}$ & $\begin{array}{l}1 \\
1\end{array}$ & $\begin{array}{l}0,29 \\
0,82\end{array}$ & $\begin{array}{l}0,34 \\
0,92\end{array}$ \\
\hline
\end{tabular}

Fonte: Autores (2021).

\section{Discussão}

A ERMAC representa uma das formas de tratamento para os casos DMT em indivíduos com maturidade esquelética (Gonçales, 2011; Chrcanovic \& Custódio, 2009; Assis et al, 2014; Bell \& Epker, 1976; Oliveira et al, 2016). Os estudos atuais buscam entender a movimentação dos movimentos expansivos da maxila, não existindo um consenso sobre qual o melhor tipo de técnica cirúrgica a ser utilizada (Betts, 2016; Bezerra, 2014; Betts et al , 1995; Gonçales, 2011; Bell \& Epker, 1976; Oliveira et al, 2016; Chung \& Goldman, 2004; Glassman et al., 1984) A ERMAC tem como principais objetivos diminuir as áreas de resistência da maxila para que seja possível uma expansão simétrica das maxilas (Bezerra, 2014; Gonçales, 2011; Bays \& Greco, 1992; Bell \& Epker, 1976) com menor taxas de movimentações dentárias ( Cureton \& Cuenin, 1999; Assis et al, 2014). Dessa forma, a literatura sugere que a técnica cirúrgica contemple a osteotomia do pilar zigomático-maxilar, pilar canino (Betts, 2016; Assis et al, 2013; Gonçales, 2011; Assis et al, 2014; Strömberg \& Holm, 1995), além da separação da placa pterigóide do osso esfenóide (Betts, 2016; Assis et al, 2013; Cureton \& Cuenin, 1999; Assis et al, 2014; Shetty et al, 1994) e a osteotomia da sutura palatina mediada (Betts, 2016).

De acordo com Assis et al (2013), a associação entre a separação da placa pterigóide do esfenóide e a osteotomia com degrau no pilar zigomático-maxilar resulta diminuição das tensões sobre o osso esfenóide e do deslocamento dentário dos dentes suportes do aparelho. Porém, um estudo de elementos finitos demonstrou que essa mesma técnica pode estar relacionada com maior dissipação de tensão aos dentes pilares (Gonçales, 2011), ao contrário de um estudo com metodologia semelhante que apontou para uma menor dissipação de forças na maxila ao utilizar um degrau no pilar zigomático-maxilar, associado com a separação placa pterigóide do osso esfenóide, o que faz com que os autores apontem essa osteotomia como a mais indicada (Assis et al, 2014).

A confecção de um degrau na parede lateral da maxila deveria diminuir a movimentação pendular da maxila, desde que esse seja realizado com o dimensão que se pretende expandir as maxilas (Betts, 2016; Betts et al, 1995). No entanto, os 
dados observados nesse estudo concordam com Oliveira et al (2016), uma vez que a presença do degrau no pilar zigomáticomaxilar parece não influenciar nos efeitos da ERMAC, sendo observado aumento significativo das distâncias maxilares para as duas técnicas, uma vez que quase todos os parâmetros maxilares apresentaram aumento em suas medidas nas comparações entre o pré-operatório (T0) e os controles pós-operatórios (T1 e T2), com exceção da largura da base óssea maxilar e da distância entre os processos alveolares palatinos no grupo II, que embora tenham aumentado, não apresentou significância estatística $(\mathrm{p}>0,05)$. Esse estudo confirmou também que, para ambos os grupos, ocorreu movimentação dos dentes anteriores e posteriores, apesar de tais medidas terem diminuído no período de contenção após a estabilização do dispositivo expansor.

A expansão simétrica de maxila representa um desafio para esse tipo de tratamento (Oliveira et al,2016). De acordo com Chamberland e Proffit (2008), uma expansão simétrica é observada quando a expansão dos molares e primeiros prémolares é semelhante. Em relação ao aumento da distância entre as maxilas, a literatura não apresentou um consenso. $\mathrm{O}$ valor do aumento da distância entre as maxilas varia entre os trabalhos: 1,31mm (Byllof \& Mossaz, 2004), 7,48mm (Chamberland \& Proffit' 2008), 4,25mm (Gurgel et al., 2014) e de 0,97mm na região anterior e 2,87 na região posterior (Oliveira et al,2016). Um levantamento da literatura apontou uma expansão do arco mandibular na ordem de 2,1mm na região dos pré-molares e de 0,25mm na região de caninos (Lagravèreet al, 2006). Porém, cabe lembrar que para cada estudo foi utilizado um tipo de técnica cirúrgica, além de diferentes formas de avaliação dessas medidas, além da variação individual, principalmente com relação à magnitude da MTD prévia ao tratamento.

O presente estudo apresenta dados concordantes com a literatura (Oliveira et al,2016; Daif, 2014), ou seja, ocorre uma expansão maior na região anterior, culminando em movimentação pendular dos segmentos, sendo comprovado por um aumento maior na região anterior de maxila para ambos os grupos, sendo de $4,16 \mathrm{~mm}$ na região anterior e de $1,5 \mathrm{~mm}$ na região posterior para o GI e de 7,6 e 1,95 mm para o GII.

Nos indivíduos representados pelo protocolo de ativação mais lento, associado à osteotomia com degrau no pilar zigomático-maxilar, foi possível observar uma expansão mais simétrica, semelhante a outros estudos (Oliveira et al,2016; Chamberland \& Proffit, 2008; Byllof et al, 2004). Nossos dados são comprovados pelo aumento estatisticamente significante, sendo esse maior na região anterior, mas ocorrendo também na região média e posterior, estando de acordo com outros trabalhos da literatura (Oliveira et al,2016; Byllof et al, 2004; Gurgel et al, 2014; Daif, 2014).

A ERMAC pode estar relacionada com vestibularização dos dentes pilares na direção do movimento expansivo (Gonçales, 2011; Assis et al, 2014; Starnbach et al, 1966; Chung \& Goldman, 2004), decorrente da transmissão de forças para os dentes pilares, resultante da ativação do aparelho expansor (Bezerra, 2014; Gonçales, 2011; Koudstaal et al, 2005; Assis et al, 2014; Starnbach et al, 1966; Oliveira et al, 2016; Garib et al., 2006; Hino et al., 2008; Loddi et al., 2008 ), sendo observada a vestibularização dos dentes superiores (Assis et al, 2013; Assis et al, 2014; Chamberland \& Proffit, 2008), rotação dos dentes pilares, deslocamento mésio-vestibular dos pré-molares e o movimento disto-bucal dos molares (Assis et al, 2014). Os dados do presente estudo revelaram que, embora tenha ocorrido um aumento da distância entre as maxilas, observou-se também aumento da angulação dentária, evidenciando a vestibularização dos mesmos, sendo esses dados observados de forma significante $(\mathrm{p}<0,05)$, independentemente da técnica cirúrgica utilizada (Assis et al, 2014; Chung \& Goldman, 2004; Oliveira et al, 2016; Gurgel et al, 2014; Daif, 2014). Uma vez que a movimentação dentária é esperada após a ativação do aparelho expansor, torna-se necessário o conhecimento da quantidade de expansão alcançada no período T2 e a quantificação da vestibularização, para que seja possível entender os resultados do tratamento.

Esses dados corroboram com o trabalho de Assis et al (2014), no qual a associação de separação da placa pterigóide e a osteotomia com degrau no pilar zigomático-maxilar não diminuem as alterações dentárias, uma vez que observamos inclinação vestibular dos dentes de suporte em ambos os grupos. Outra possível explicação para a maior vestibularização nos indivíduos do grupo II pode ser a ativação lenta do aparelho expansor, ao contrário do grupo I, na qual não existiu um intervalo 
de tempo após 7 dias de ativação do aparelho expansor. Como forma de evitar essa complicação, a literatura sugere a associação entre ERMAC e distratores palatinos ósseo-suportados, uma vez que esses apresentam apoio próximo a região de resistência óssea, sem exercer força nos dentes de suporte (Koudstaal et al, 2005; Garibet al, 2006).

A expansão da maxila pode estar relacionada com elevadas taxas de recidiva (Strömberg \& Holm, 1995; Chamberland \& Proffit, 2008), as quais podem ser de 30\% (Chamberland \& Proffit, 2008) a 36\% (Byllof \& Mossaz, 2004), sendo a retenção ortodôntica responsável pela estabilidade pós-operatória (Chamberland \& Proffit, 2008), uma vez que pode ocorrer redução da largura do arco após esse período, especialmente na região dos dentes pilares do aparelho (Oliveira et al.,). $\mathrm{Na}$ literatura analisada, observou-se que mesmo a utilização de contenção ortodôntica por um período de 3 a 6 meses (Strömberg \& Holm, 1995; Byllof \& Mossaz, 2004) relaciona-se com recidiva dentária e esquelética, assim como com os nossos resultados.

No presente estudo, embora tenham ocorrido alterações dimensionais no período de contenção (T2-T1), a maioria delas não apresentou significância estatística. No grupo I observou-se uma diminuição da vestibularização dos dentes 13 e 23 e do ângulo do dente 23, sugerindo uma possível verticalização desses dentes, com provável torque sobre esse dente, com movimentação maior do ápice no sentido vestibular.

Na região posterior, ambos os grupos apresentaram diminuição significante $(\mathrm{p}=0,00)$ da distância entre os ápices dos molares, o que possivelmente sugere uma movimentação dentária que não é estabilizada pelo aparelho quando este permanece sem ativação, ao contrário dos dados apresentados por Oliveira et al (2016). Essa diferença de resultados pode ser explicada pelo fato que os estudos apresentam metodologias distintas. No presente estudo, a mensuração das resultantes após a ERMAC foi realizada baseando-se no longo eixo dentário, dessa forma apresentando um melhor entendimento da real movimentação dentária.

A diminuição das medidas observadas em ambos os grupos entre os períodos T1 e T2, podem ser explicadas pela influência de ações musculares, estabilização oclusal, início do tratamento ortodôntico após a remoção do expansor, resistência e recidiva dentária por conta do ligamento periodontal, ação do dispositivo expansor ou pela presença da barra palatina (Bezerra, 2014; Oliveira et al, 2016). Em um estudo conduzido por Gurgel et al (2014), os autores relatam que após a contenção, apenas a região inter-incisivos foi significativamente diminuída. A taxa de recidiva foi maior nos indivíduos do grupo I, o que sugere que a corticotomias em forma de degrau na região do pilar zigomático-maxilar, não exerce influência sobre esse parâmetro.

Os indivíduos submetidos à expansão ortopédica de maxila (ERM) podem evoluir com extrusão e inclinação dos superiores, contribuindo para a mudança de posicionamento mandibular (Bishara \& Staley, 1987), traduzida por avanço e rotação horária, porém sem concordância da magnitude e permanência desse movimento (Bishara \& Staley, 1987), podendo apresentar-se como uma situação transitória (Oliveira et al, 2017). A literatura aponta a possibilidade de rotação mandibular no sentido horário (Günbay eta al, 2008; Atac et al, 2006; Lineberger et al., 2012), mas de acordo com o estudo de Oliveira et al (2017), não são observadas alterações após 6 meses.

De acordo com McNamara (2000), os efeitos da ERM também podem ser observados no arco dentário inferior, ocorrendo um alargamento do mesmo devido, principalmente, à "descompensação" e encaixe dos dentes posteriores inferiores, os quais podem estar lingualizados como resultado da atresia maxilar. Dessa forma, a ERM pode resultar ainda em alterações nas distâncias entre os caninos e os molares superiores e inferiores, podendo esse procedimento influenciar na posição dos dentes inferiores (Bishara \& Staley, 1987), resultando em aumento do arco mandibular nos indivíduos submetidos a esse tipo de tratamento (Lineberger et al, 2012; McNamara et al., 2003; Bacetti et al., 2001). 
Embora a ERMAC possa se relacionar com diminuição do SNB (Atac et al, 2006) e rotação anti-horária da mandíbula (Oliveira et al, 2017), não existem estudos que comprovem a movimentação dos dentes inferiores, uma vez que os movimentos dos molares inferiores se apresentam sem significância estatística (Chamberland \& Proffit, 2008).

Nesse estudo foram observadas discretas alterações na largura lingual, distância entre ápices e inclinação vestibular dente 46. Contudo, esses dados não foram estatisticamente significantes ( $\mathrm{p}>0,05$ ). A única medida com alteração significante foi a angulação dos dentes 36, sendo observado um aumento nos períodos T0-T1 para ambos os grupos, e um aumento de T0T2 no grupo I. Esses dados demonstram que praticamente não ocorrem efeitos dento-esqueléticos mandibulares para os parâmetros estudados, após a ERMAC. Porém, a espessura da cortical lingual no dente 46 mostrou redução, além do aumento das distâncias entre os ápices dos dentes 36 e 46, ambos provavelmente decorrentes da inclinação vestibular do dente 46.

Uma possível explicação para o aumento da distância entre os ápices dos dentes inferiores seria a ocorrência de contato prematuro dos dentes inferiores com os dentes superiores, agora em uma nova posição, ou seja, mais vestibularizada. Outra possível explicação para serem observadas alterações somente no dente 36, relaciona-se ao fato da maioria dos indivíduos apresentar maior carga oclusal no lado direito, sendo esse considerado como "lado de trabalho".

Frente aos dados obtidos nesse estudo, podemos destacar que ERMAC não resulta em alterações estatisticamente significantes para com relação ao posicionamento dos dentes inferiores.

Esses dados são importantes, uma vez que os dentes superiores e inferiores são os parâmetros para observar a correção da DMT, orientando para a manutenção ou não do aparelho expansor (Albuquerque, Tieghi-Neto, Nogueira \& Gonçales, 2013). O planejamento pré-operatório deve considerar a possibilidade de evitar torque lingual das coroas dos molares superiores e/ou torque vestibular dos molares inferiores, evitando-se recidiva da mordida cruzada posterior (Bishara \& Staley, 1987). Dessa forma, é possível que a presença de contatos pré-maturos durante o movimento expansivo resulte em alterações dos dentes inferiores. De acordo com Betts (2016), os dentes inferiores deveriam ser descompensados antes da ERMAC, permitindo uma avaliação da quantidade de expansão transversal necessária, minimizando os riscos de recidiva e evitar sobre-correções excessivas ou sub-correções da deficiência transversal de maxila. Salienta que a adoção de diferentes parâmetros, bem como mais pesquisas sobre os efeitos dento-esqueléticos mandibulares da ERMAC podem se tornar uma necessidade.

\section{Conclusão}

A realização do degrau no pilar zigomático-maxilar não diminui a movimentação dentária na maxila. Para os dentes inferiores, embora sem significância estatística, a movimentação destes pode ser atribuída à presença de contatos prematuros após correção da deficiência maxilar transversa.

\section{Referências}

Albuquerque, G. C., Tieghi-Neto, V., Nogueira, A. S., \& Gonçales, E. S. (2013). Complicações após expansão de maxila cirurgicamente assistida. Revista de Odontologia da UNESP (42), 20-4.

Assis, D. S. F. R., Xavier, T. A., Noritomi, P. Y., Gonçales, A. G. B., Ferreira, Jr O., Carvalho, P. C. P., \& Gonçales, E. S. (2013). Finite element analysis of stress distribution in anchor teeth in surgically assisted rapid palatal expansion. Int. J. Oral Maxillofac. Surg (42) 1093-9.

Assis, D. S. F. R., Xavier, T. A., Noritomi, P. Y., \& Gonçales. (2014). Finite Element Analysis of Bone Stress After SARPE. J Oral Maxillofac Surg. 72(167), 1-7.

Atac, A. T. A., Karasu, H. A., \& Aytac, D. (2016). Surgically assisted rapid maxillary expansion compared with orthopedic rapid maxillary expansion. Angle Orthod. 76(3), 353-9.

Bacetti, T., Franchi, L., Cameron, C. G., \& McNamara, J. A. (2001). Treatment Timing for Rapid Maxillary Expansion. Angle Orthod (71), 343-50. 
Bays, R. A., \& Greco, J. N. (1992). Surgically Assisted Rapid Palatal Expansion: an outpatient technique with long-term stability. J Oral Maxillofac Surg (50), $110-3$.

Bell, W. H., \& Epker, B. N. (1976). Surgical-orthodontic expansion of the maxilla. Am J Orthod. (70), 517-28.

Betts, N. J., Vanarsdall, R. L., Barber, H. D., Higgins-Barber, K., \& Fonseca, R. J. (1995). Diagnosis and treatment of transverse maxillary deficiency. Int J Adult Orthod Orthognath Surg10(2), 75-96.

Betts, N. J. (2016). Surgically Assisted Maxillary Expansion. Atlas Oral Maxillofac Surg Clin N Am 24(1), 67-77.

Bezerra, M. F. (2014). Efeitos dento-esqueléticos da expansão rápida de maxila assistida cirurgicamente com e sem disjunção pterigomaxilar. (Tese de Doutorado). Universidade Federal do Ceará. Fortaleza. Brasil.

Bishara, S. E., \& Staley, R. N. (1987). Maxillary expansion: Clinical implications. Am J Orthod Dentofac Orthop (91) 3-14.

Byllof, K., \& Mossaz, F. (2004). Skeletal and dental anges following surgically assisted rapid palatal. Eur J Orthod. (26), 403-9.

Chamberland, S., \& Proffit, W. R. (2008). Closer Look at the Stability of Surgically Assisted Rapid Palatal Expansion. J Oral Maxillofac Surg (66), 1895-900.

Chrcanovic, B. R., \& Custódio, A. L. N. (2009). Orthodontic or surgically assisted rapid maxillary expansion. Oral Maxillofac Surg (13), 123-37.

Chung, C. H., \& Goldman, A. M. (2004). Dental tipping and rotation immediately after surgically assisted rapid palatal expansion. Eur J Orthod. (25), 353-8.

Cureton, S. L., \& Cuenin, M. (1999). Surgically assisted rapid palatal expansion: Orthodontic preparation for clinical success. Am J Orthod Dentofacial Orthop (116), 46-59.

Daif, E. T. (2014). Segment tilting associated with surgically assisted rapid maxillary expan- sion. Int J Oral Maxillofac Surg (43), 311-5.

Garib, D. C., Henriques, J. F., Janson, G., Freitas, M. R., \& Fernandes, A. Y. (2006). Periodontal effects of rapid maxillary expansion with tooth-tissue borne and tooth-borne expanders: a computed tomography evaluation. Am J Orthod Dentofacial Orthop (129), 749-58.

Glassman, A. S., Nahigian, S. J., Medway, J. M., \& Aronowitz, H. I. (1984). Conservative surgical orthodontic adult rapid palatal expansion: sixteen cases. Am J Orthod (86), 207-13.

Gonçales, E. S. (2011). Análise da distribuição das tensões dentárias em maxila submetida à expansão cirurgicamente assistida. (Tese para obtenção do título de Livre Docente em Cirurgia e Traumatologia Bucomaxilofaciais). Faculdade de Odontologia de Bauru, da Universidade de São Paulo, Bauru, Brasil.

Gijt, J. P., Gül, A., Tjoa, S. T. H., Wolvius, E. B., van der Wal, K. G. H., \& Koudstaal, M. J. (2017). Follow up of surgically-assisted rapid maxillary expansionafter 6.5 years: skeletal and dental effects. Br J Oral Maxillofac Surg (55), 56-60.

Günbay, T., Akay, M. C., Günbay, S., Aras, A., Koyuncu, B. O., \& Sezer, B. (2008). Transpalatal distraction using bone-borne distractor: clinical observations and dental and skeletal changes. J Oral Maxillofac Surg 66(12), 2503-14.

Gurgel, J. A., Tiago, C. M., \& Normando, D. (2014). Transverse changes after surgically assisted rapid palatal expansion. Int J Oral Maxillofac Surg 43(3), $316-22$.

Hino, C. T., Pereira, M. D., Sobral, C. S., Kreniski, T. M., \& Ferreira, L. M. (2008). Transverse effects of surgically assisted rapid maxillary expansion: a comparative study using Haas and Hyrax. J Craniofac Surg (19), 718-25.

Koudstaal M. J, Poort L. J, van der Wal K. G, Wolvius E. B, Prahl-Andersen B, \& Schulten A. J. (2005). Surgically assisted rapid maxillary expansion (SARME): a review of the literature. Int J Oral Maxillofac Surg 34(7), 709-14.

Lagravère M. O, Major P. W, \& Flores-Mir C (2006). Dental and skeletal changes following surgically assisted rapid maxillary expansion. Int J Oral Maxillofac Surg 35(6), 481-7.

Lineberger M. W, McNamara J. A, Baccett T,Herberger T, \& Franchi L. (2012). Effects of rapid maxillary expansion in hyperdivergent patients. Am J Orthod Dentofacial Orthop (142), 60-9.

Loddi P, Pereira M. D, Wolosker A. B, Hino C T, Kreniski T. M, \& Ferreira L. M. (2008). Transverse effects after surgically assisted rapid maxillary expansion in the midpalatal suture using computed tomography. J Craniofac Surg (19), 433-8.

McNamara J. A. (2000). Maxillary transverse deficiency. Am J Orthod Dentofacial Orthop 117(5), 567-70.

McNamara J. A., Bacetti T., Franchi L., \& Herberger T. A. (2003). Rapid Maxillary Expansion Followed by Fixed Appliances: A Long-term Evaluation of Changes in Arch Dimensions. Angle Orthod (73), 344-53.

Melsen B. (1972). A histological study of the influence of sutural morphology and skeletal maturation on rapid palatal expansion in children. Trans Eur Orthod Soc, 499-507.

Oliveira T. F. M., Pereira-Filho V. A., Gabrielli M. A. C., Gonçales E. S., \& Santos- Pinto A. (2016). Effects of lateral osteotomy on surgically assisted rapid maxillary expansion. Int. J. Oral Maxillofac. Surg (45), 490-6.

Oliveira T. F. M., Pereira-Filho V. A. M. Gabrielli M. F. R., Gonçales E. S, \& Santos-Pinto A. (2017). Effects of surgically assisted rapid maxillary expansion on mandibular position: a three dimensional study. Progress in Orthodontics,18-22.

Proffit W. R, Turvey T. A, \& Phillips C. (1996). Orthognathic surgery: a hierarchy of stability. Int J Adult Orthodon Orthognath Surg 11(3), 191-204. 
Research, Society and Development, v. 11, n. 1, e2811124152, 2022

(CC BY 4.0) | ISSN 2525-3409 | DOI: http://dx.doi.org/10.33448/rsd-v11i1.24152

Seeberger R, Abe-Nickler D, Hoffmann J, Kunzmann K, \& Zingler S (2015). One-stage tooth-borne distraction versus two stage bone-borne distraction in surgically assisted maxillary expansion (SARME). Oral Surg Oral Med Oral Pathol Oral Radiol (120), 693-8.

Silverstein K, \& Quinn P. D. (1997). Surgically-Assisted Rapid Palatal Expansion for Management of Transverse Maxillary Deficiency. J Oral Maxillofac Surg (55), 725-7.

Shetty V, Caridad J. M, Caputo A. A, \& Chaconas S. J. (1994). Biomechanical Rationale for Surgical-Orthodontic Expansion of the Adult Maxilla. J Oral Maxillofac Surg (52), 742-9.

Starnbach, H (1996). Facioskeletal and dental changes resulting from rapid maxillary expansion. Ang Orthod (36), 152-164.

Strömberg C, \& Holm J (1995). Surgically assisted, rapid maxillary expansion in adults. A retrospective long-term follow-up study. J Craniomaxillofac Surg (23), 222- 7

Suri L, \& Taneja P (2008). Surgically assisted rapid palatal expansion: a literature review. Am J Orthod Dentofacial Orthopm133(2), 290-302.

Sygouros A, Motro M, Ugurlu F, \& Acard A (2014). Surgically assisted rapid maxillary expansion: Cone-beam computed tomography evaluation of different surgical techniques and their effects on the maxillary dentoskeletal complex. Am J Orthod Dentofacial Orthop (146), 748-57.

Vandersea B. A, Ruvo A. T, \& Frost D. E. (2007). Maxillary transverse deficiency - Surgical Alternatives to management. Oral Maxillofac Clin N Am (19), 351-68. 